Review Article

\title{
Mini Review: Anticholinergic Activity as a Behavioral Pathology of Lewy Body Disease and Proposal of the Concept of "Anticholinergic Spectrum Disorders"
}

\author{
Koji Hori, ${ }^{1,2}$ Kimiko Konishi, ${ }^{3}$ Misa Hosoi, ${ }^{2}$ Hiroi Tomioka, ${ }^{2}$ Masayuki Tani, ${ }^{4}$ \\ Yuka Kitajima, ${ }^{5}$ and Mitsugu Hachisu ${ }^{6}$ \\ ${ }^{1}$ Department of Neuropsychiatry, St. Marianna University, School of Medicine, Kanagawa, Japan \\ ${ }^{2}$ Department of Psychiatry, Showa University Northern Yokohama Hospital, Kanagawa, Japan \\ ${ }^{3}$ Tokyo Metropolitan Tobu Medical Center for Persons with Developmental/Multiple Disabilities, Tokyo, Japan \\ ${ }^{4}$ Department of Psychiatry, Showa University East Hospital, Tokyo, Japan \\ ${ }^{5}$ Department of Anesthesiology, School of Medicine, Juntendo University, Tokyo, Japan \\ ${ }^{6}$ Department of Pharmaceutical Therapeutics, Division of Clinical Pharmacy, School of Pharmacy, Showa University, Tokyo, Japan
}

Correspondence should be addressed to Koji Hori; kojihori@med.showa-u.ac.jp

Received 7 May 2016; Accepted 26 July 2016

Academic Editor: Per Odin

Copyright (C) 2016 Koji Hori et al. This is an open access article distributed under the Creative Commons Attribution License, which permits unrestricted use, distribution, and reproduction in any medium, provided the original work is properly cited.

\begin{abstract}
Given the relationship between anticholinergic activity (AA) and Alzheimer's disease (AD), we rereview our hypothesis of the endogenous appearance of $\mathrm{AA}$ in $\mathrm{AD}$. Briefly, because acetylcholine (ACh) regulates not only cognitive function but also the inflammatory system, when ACh downregulation reaches a critical level, inflammation increases, triggering the appearance of cytokines with AA. Moreover, based on a case report of a patient with mild AD and slightly deteriorated ACh, we also speculate that AA can appear endogenously in Lewy body disease due to the dual action of the downregulation of ACh and hyperactivity of the hypothalamic-pituitary-adrenal axis. Based on these hypotheses, we consider AA to be a behavioral pathology of Lewy body disease. We also propose the concept of "anticholinergic spectrum disorders," which encompass a variety of conditions, including $\mathrm{AD}$, Lewy body disease, and delirium. Finally, we suggest the prescription of cholinesterase inhibitors to patients in this spectrum of disorders to abolish AA by upregulating ACh.
\end{abstract}

\section{Introduction}

The neurotransmitter acetylcholine (ACh) and anticholinergic activity (AA) can bind to the muscarinic acetylcholine receptor [1]. AA includes every substance that binds the muscarinic acetylcholine receptor; however, generally speaking $\mathrm{AA}$ antagonizes $\mathrm{ACh}$ to the muscarinic acetylcholine receptor. Therefore, AA disturbed the function of ACh. AA predominantly disturbs memory function $[2,3]$, with psychotic symptoms more prominent than cognitive dysfunctions $[4,5]$. The main cause of AA is prescribed medication [6], but physical illness [7] and mental stress which cause elevated cortisol [8] also cause AA. We previously reported that AA was also caused by ACh downregulation and proposed our hypothesis of the endogenous appearance of AA in Alzheimer's disease (AD) $[9,10]$. That is, because ACh regulates not only cognitive function but also the inflammatory system, when ACh downregulation reaches a critical level, inflammation is increased, triggering the appearance of cytokines with AA. These processes might also accelerate $\mathrm{AD}$ pathologies $[9,10]$. Moreover, we speculated that, in addition to $\mathrm{AD}$, other neurocognitive disorders such as Lewy body disease (LBD) and delirium are related to AA and that the appearance of $\mathrm{AA}$ in $\mathrm{LBD}$ is also related to an endogenous factor [11]. In this article, we rereview our hypotheses surrounding the endogenous appearance of AA in $\mathrm{AD}[9,10]$ and LBD [11]. We also theorize that the onset of clinical symptoms in LBD depends on the endogenous appearance of AA in LBD and propose a new concept of "anticholinergic spectrum disorders." 


\section{Hypothesis of Endogenous Anticholinergic Activity in Alzheimer's Disease}

We previously evaluated the relationship between clinical symptoms and AA in $76 \mathrm{AD}$ patients [12]. Serum anticholinergic activity (SAA), a peripheral marker of anticholinergic burden, was positive in 26 of these patients; the other 50 were negative for SAA. Delusions, hallucinations, diurnal rhythm disturbances, and global cognitive dysfunctions were significantly more severe in the SAA-positive group than in the SAA-negative group, and the patients in the SAApositive group took more psychotropic medicines. Moreover, SAA positivity was more related to psychotic symptoms than cognitive dysfunctions. Our results indicated that clinical symptoms and prescription of psychotropic medicines are factors related to SAA, particularly psychotic symptoms. Because these results were from a cross-sectional study, we could not elucidate the causal relationships among these three factors (psychotic symptoms, the prescription of psychotropic medicines, and SAA). However, we speculated that there might be a cyclic relationship among the factors. Accordingly, we named this association the "vicious cycle of anticholinergic activity in AD (VCAA)" [12].

Positive SAA can be caused by various medicines and AA worsens clinical psychiatric symptoms. However, psychotropic medicines are generally prescribed for the clinical psychiatric symptoms of agitation and psychosis in $\mathrm{AD}[13,14]$. Therefore, the relationship among prescribed psychotropic medicines, SAA, and clinical symptomsespecially hallucinations and a disturbed diurnal rhythmmight be cyclic. Moreover, because psychotropic medicines are typically prescribed for the clinical psychiatric symptoms of agitation and psychosis in $\mathrm{AD}[13,14]$, psychotropic medicines might not be the first step in this cycle. There might be a causal relationship between the other two factors comprising the VCAA, namely, psychotic symptoms and the use of psychotropic medicines, with AA worsening psychiatric symptoms. Therefore, we consider the appearance of AA to be the first step in the VCAA: that is, AA appears endogenously in AD [12]. In fact, there is a high probability that the pathogenesis of $\mathrm{AD}$ involves neuronal degeneration due to oxidative stress, and it has been shown that amyloid might be able to generate free radicals [15]. On the other hand, an endogenous ligand of the muscarinic receptor is found to a greater extent in the $\mathrm{AD}$ brain than in the nondemented control brain, and the endogenous ligand of the muscarinic receptor seems to be a low-molecular weight substance of $100-1,000 \mathrm{Da}$ that is catalyzed by oxidation [16]. Thus, SAA might not always derive purely from prescribed psychotropic medicines; it may also derive from endogenous oxidative products [12].

In short, we hypothesized that the relationship among psychotic symptoms, positive SAA, and the prescription of psychotropic medicines is a cyclic one and that AA appears endogenously in $\mathrm{AD}[12]$, because psychotropic medicines are prescribed for psychotic symptoms [13, 14]. We also speculated about the mechanism behind the endogenous appearance of $\mathrm{AA}$ in $\mathrm{AD}[9,10]$. ACh regulates not only cognitive function but also the inflammatory system $[17,18]$.
Therefore, we surmised that because AD is characterized by the downregulation of $\mathrm{ACh}$, the inflammatory system is upregulated in $\mathrm{AD}$ when the level of $\mathrm{ACh}$ reaches a specific threshold (i.e., moderately severe disease) $[9,10]$. Thus, downregulation of ACh would cause downregulation of the anti-inflammatory pathway (the cholinergic anti-inflammatory pathway), permitting upregulation of the inflammatory pathway $[17,18]$. The hyperactive inflammation generates cytokines with AA, such as C-reactive protein [19].

Moreover, stimulation of muscarine 1 receptor is favorable for reducing amyloid pathology [20, 21]. Therefore, we considered that AA accelerates amyloid pathology. In fact, anticholinergic loads are reported to increase amyloid pathology [22, 23]. We thus proposed the hypothesis of the endogenous appearance of $\mathrm{AA}$ in $\mathrm{AD}$.

\section{Dual Actions of Anticholinergic Inserts Cause Anticholinergic Activity and the Endogenous Appearance of Anticholinergic Activity in Lewy Body Disease}

We then encountered a 74-year-old woman with positive SAA, although her cognitive decline was not at a sufficiently critical level to elicit endogenous AA [24]. In this instance, we hypothesized that the SAA positivity was induced by the addition of mental stress to a preexisting ACh downregulation [24]. We consider mental stress and other factors besides ACh downregulation to be capable of inducing SAA, to be socalled "AA inserts." In this context, because there are other AA inserts besides ACh downregulation $[9,10]$, such as the prescribed medication [6], physical illness [7], and mental stress [8], dual actions of AA inserts can also cause AA when the ACh level does not reach a critical level (i.e., at the stage of mild cognitive impairment or at a mild stage of the disease) [24]. Based on this case, we speculated that the appearance of AA might cause psychiatric symptoms such as delusions, hallucinations, and diurnal rhythm disturbances in delirium and LBD based on the dual actions of ACh downregulation [11] (if ACh is not deteriorated or overloaded, the intact $\mathrm{ACh}$ system can be upregulated and compensate for another AA insert [10]) and the effects of another AA insert.

We also previously evaluated the relationship between postoperative delirium and SAA [11]. Although delirium is considered an important issue among elderly patients in various settings, the mechanism underlying delirium is poorly understood [25]. We concluded that delirious patients fail to compensate for the increase in AA, making it important to pay close attention to the perioperative transition of the SAA level in relation to delirium, rather than focusing on a single SAA level [11].

The factor suggesting the endogenous appearance of AA in LBD is the dual action of a deteriorated autonomic parasympathetic nervous system and a relatively minor decrease in ACh [11]. This is attributable to the induction of $\mathrm{AA}$ in $\mathrm{LBD}$, with the deteriorated autonomic parasympathetic nervous system increasing the activity of the hypothalamic-pituitary-adrenal (HPA) axis [26] and hypercortisolism [27, 28]. If inflammatory processes are caused 
by AA, it is plausible to conclude that corticosteroids would inhibit the action of AA because of their anti-inflammatory properties.

Nonetheless, corticosteroids have been reported to induce or increase AA in the brain [8] and cause delirium [25]. Typically, the corticosteroid level in plasma is high early in the morning and rapidly declines thereafter. We theorized that this rapid reduction might cause immune system disinhibition, with immune system activation in the afternoon, evening, and night [10], because cortisol levels after awakenings, such as in the morning, might be affected in neurocognitive and neuropsychiatric disorders [10]. If the blood level of corticosteroids rises above normal, the subsequent decline in the level can be expected to be greater, where it is expected that the inflammatory state activated the AA. Therefore, it seems logical that even if AA does not appear early in the morning, it may appear by noon or later. This mechanism might explain why patients with delirium can appear calm in the morning but delirious in the late afternoon and at night (i.e., sundowning) [10]. We also believe that deteriorated parasympathetic autonomic nervous function causes AA in patients with $\mathrm{LBD}$ based on the small degree of downregulation of $\mathrm{ACh}$ and that $\mathrm{AA}$ appears endogenously in LBD [11].

Briefly, AA appears earlier in LBD than in AD because of the combination of HPA axis hyperactivity and the small degree of ACh downregulation. In delirium, AA appears, as in LBD, due to the combination of these two factors. In LBD, HPA axis hyperactivity occurs endogenously by way of dysfunction of the parasympathetic nervous system [27, 28]. In contrast, HPA axis hyperactivity occurs exogenously in delirium due to mental stress and/or physical illnesses. AA is thus related to the pathogenesis of $\mathrm{AD}, \mathrm{LBD}$, and delirium.

We can explain the onset of clinical symptoms using our hypothesis of the endogenous appearance of AA in LBD. This hypothesis is shown in Figure 1. Briefly, based on a small degree of ACh downregulation, HPA axis hyperactivity caused by a deteriorated autonomic parasympathetic nervous system gradually worsens and finally induces hyperactive inflammation, which also causes AA. Continuous and recurrent appearance of AA exacerbates the amyloid pathology and further downregulates $\mathrm{ACh}$. We divided this entire process into the following three stages, in this order: (1) deterioration of the parasympathetic nervous system (and hyperactivity of the HPA axis); (2) appearance of AA; and (3) downregulation of $\mathrm{ACh}$ caused by continuous and recurrent appearance of AA.

At the stage of parasympathetic nervous system deterioration, which corresponds to the prodromal stage of LBD, HPA axis hyperactivity gradually worsens but does not reach the level at which AA is induced. Therefore, symptoms related to dysfunction of the parasympathetic nervous system occur in LBD, such as REM behavioral symptoms, syncope, and constipation [29]. Moreover, depression, which is related to HPA axis hyperactivity, also develops (Figure 2). We have already reported that anxiety and affective disturbances in $\mathrm{AD}$ patients are connected to delusion, hallucination, and aggressiveness by aging and the disease progress [30]. Therefore, if the degree of ACh dysfunction is relatively

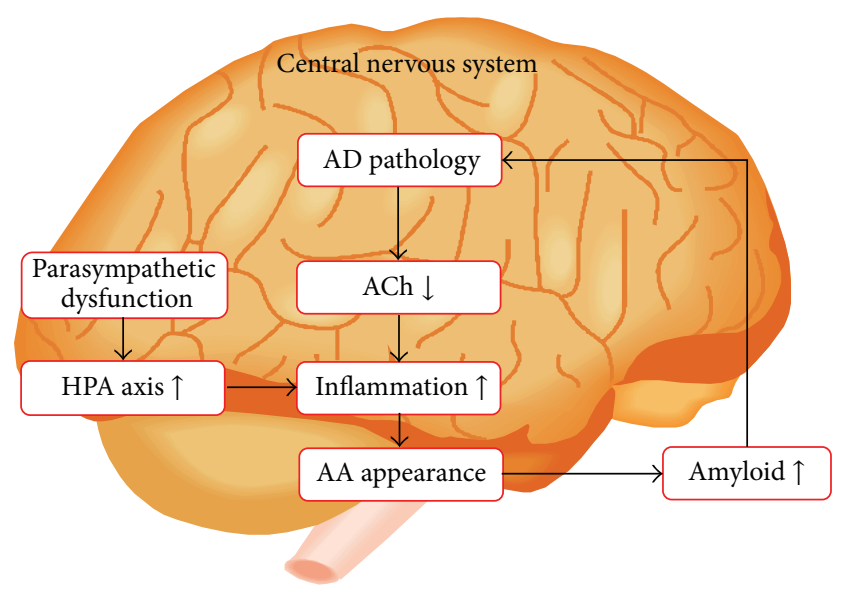

FIgUre 1: AA in LBD appears earlier than in Alzheimer's disease because of a combination of HPA axis hyperactivity and a small degree of ACh downregulation. In LBD, HPA axis hyperactivity occurs endogenously due to dysfunction of the parasympathetic nervous system. We refer to these processes as the hypothesis of the endogenous appearance of AA in LBD. Based on the small degree of ACh downregulation, HPA axis hyperactivity caused by deterioration of the autonomic parasympathetic nervous system gradually worsens before finally inducing hyperactive inflammation, which also causes AA. Continuous and recurrent appearance of AA exacerbates the amyloid pathology and further downregulates ACh. The order of the process is as follows: (1) deterioration of the parasympathetic nervous system, (2) hyperactivity of the HPA axis, (3) appearance of AA, and (4) downregulation of ACh. AA: anticholinergic activity, ACh: acetylcholine, AD: Alzheimer's disease, HPA axis: hypothalamic-pituitary-adrenal axis, and LBD: Lewy body disease. This figure is reproduced from Hori et al. [9] with permission from the Japanese Society of Neuropsychopharmacology (Tokyo, Japan).

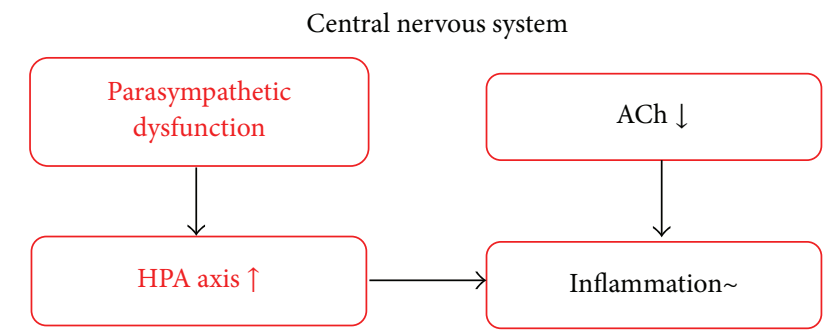

FIGURE 2: At the stage of parasympathetic nervous system deterioration, which corresponds to the prodromal stage of LBD, HPA axis hyperactivity gradually worsens but does not reach the level at which AA is induced. Therefore, symptoms related to dysfunction of the parasympathetic nervous system occur in LBD, such as REM behavioral symptoms, syncope, and constipation. Moreover, depression, which is related to HPA axis hyperactivity, also develops.

large, depression appears as behavioral and psychological symptoms of dementia in AD.

At the stage of AA appearance, which corresponds to the early stage of LBD, HPA axis hyperactivity reaches a critical level and induces AA. In the early stage of LBD, symptoms related to AA develop, including psychotic symptoms such 


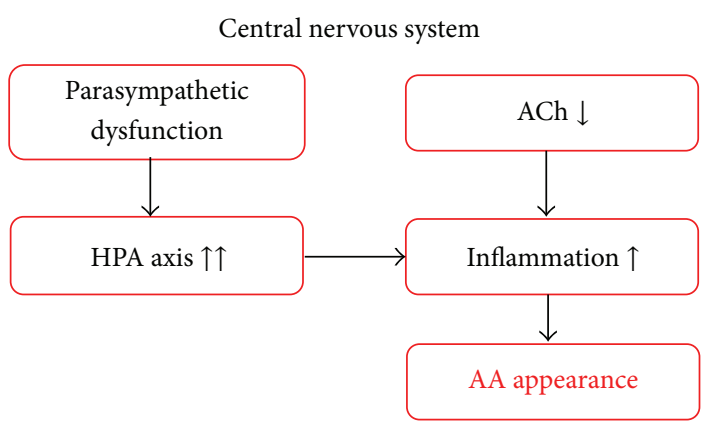

FIgURE 3: At the stage of AA appearance, which corresponds to the early stage of LBD, HPA axis hyperactivity reaches a critical level and induces AA. In the early stage of LBD, symptoms related to AA develop, including psychotic symptoms such as visual hallucinations, delusions, and diurnal rhythm disturbances. These symptoms are similar to those of delirium and are included as the core symptoms of the diagnostic criteria.

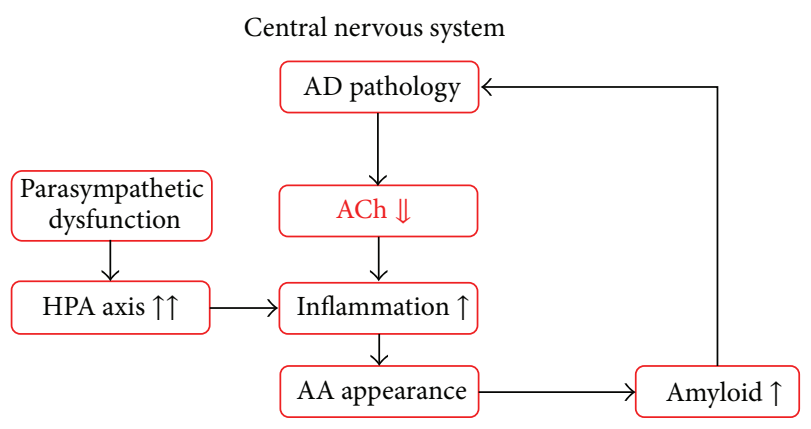

FIgURE 4: At the stage of ACh downregulation, corresponding to the late stage of $\mathrm{LBD}$, the patient develops symptoms related to ACh downregulation, which is induced by the continuous and recurrent appearance of AA. These symptoms include memory disturbances and disorientation to time and place. AA: anticholinergic activity, ACh: acetylcholine, AD: Alzheimer's disease, HPA axis: hypothalamic-pituitary-adrenal axis, and LBD: Lewy body disease.

as visual hallucinations, delusions, and diurnal rhythm disturbances. These symptoms are similar to those of delirium and are included as the core symptoms of the diagnostic criteria [29] (Figure 3). When patients show these psychotic symptoms, they are typically referred to a department of psychiatry. Similarly, patients with LBD are also generally referred to the same department at this stage, and we consider AA (psychosis) and HPA axis hyperactivity (depression), as triggers of AA, to be behavioral pathologies of LBD.

At the stage of ACh downregulation, corresponding to the late stage of LBD, the patient develops symptoms related to ACh downregulation, which is induced by the continuous and recurrent appearance of AA. These symptoms include memory disturbances and executive dysfunction [31] (Figure 4).

AA appears when the ACh deterioration reaches a critical level, that is, at a moderate stage in $\mathrm{AD}$. Therefore, $\mathrm{AA}$ is related not only to behavioral symptoms, such as delusions, hallucinations, and diurnal rhythm disturbances [12], but also to cognitive dysfunctions, such as memory disturbances and

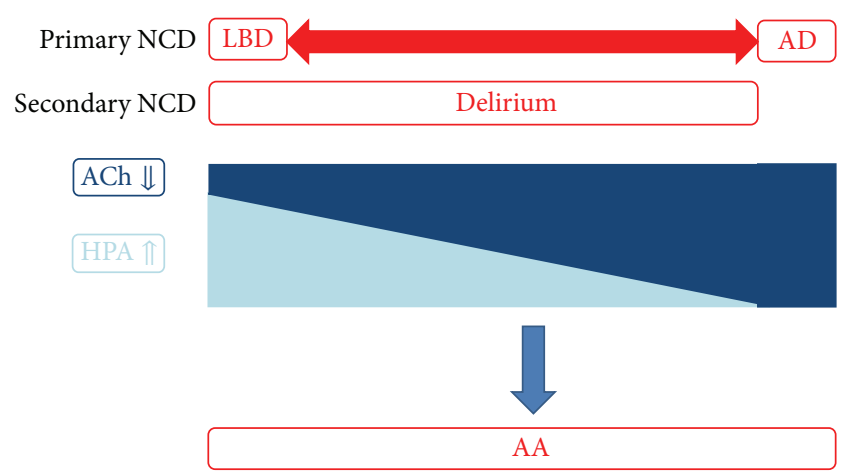

FIgURE 5: When the ACh deterioration is small, a larger AA insert of HPA axis dysfunction is necessary for the appearance of AA. However, when the ACh deterioration is large, a small AA insert of HPA axis dysfunction is sufficient for the appearance of AA. Of course, when the ACh deterioration is at a critical level, AA appears without HPA axis dysfunction. However, if ACh is not deteriorated or overloaded, the intact ACh system can be upregulated and compensate for any other AA inserts. We refer to the conditions encompassed by this concept as "anticholinergic spectrum disorders," which include $\mathrm{AD}, \mathrm{LBD}$, and delirium. AD and LBD are primary NCDs, whereas delirium is a secondary NCD. AA: anticholinergic activity, ACh: acetylcholine, AD: Alzheimer's disease, HPA axis: hypothalamic-pituitary-adrenal axis, LBD: Lewy body disease, and NCD: neurocognitive disorder.

executive dysfunction. However, the ACh deterioration in $\mathrm{LBD}$ and delirium is not as severe as that of $\mathrm{AD}$ when $\mathrm{AA}$ appears. Therefore, only behavioral symptoms are prominent in LBD and delirium, such as delusions, hallucinations, and diurnal rhythm disturbances. This is why the symptoms of LBD and delirium are similar. We consider the pathophysiology of the clinical symptoms of LBD to be related to AA.

\section{Proposal of "Anticholinergic Spectrum Disorders" in Neurocognitive Disorders}

Based on these hypotheses, we propose that certain neurocognitive disorders, such as $\mathrm{AD}, \mathrm{LBD}$, and delirium, be considered "anticholinergic spectrum disorders." In AD, AA appears endogenously when the downregulation of $\mathrm{ACh}$ reaches a critical level. In contrast, the AA in LBD and delirium involves a combination of HPA axis hyperactivity and a slight ACh downregulation. When the ACh deterioration is small, a larger AA insert of HPA axis dysfunction is necessary for the appearance of AA. On the other hand, when the ACh deterioration is large, a small AA insert of HPA axis dysfunction is sufficient. Of course, if the ACh deterioration is at a critical level, AA appears without HPA axis dysfunction. However, as previously discussed, if $\mathrm{ACh}$ is not deteriorated or overloaded, the intact ACh system can be upregulated and compensate for another AA insert. We refer to the conditions encompassed by this concept as "anticholinergic spectrum disorders" (Figure 5).

Possible pharmacotherapies for anticholinergic spectrum disorders are of course also important. Yilmaz et al. [32] described the case of a 19-year-old man with anticholinergic 


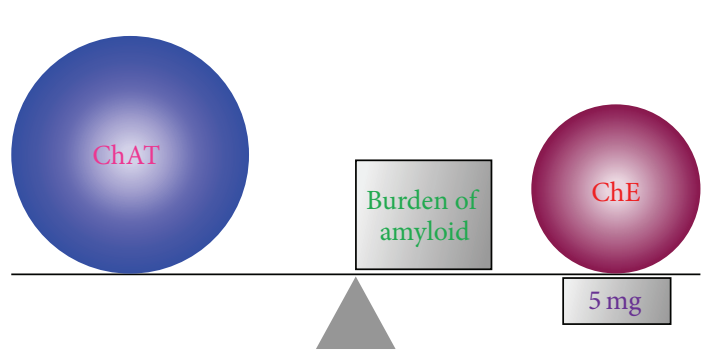

(a)

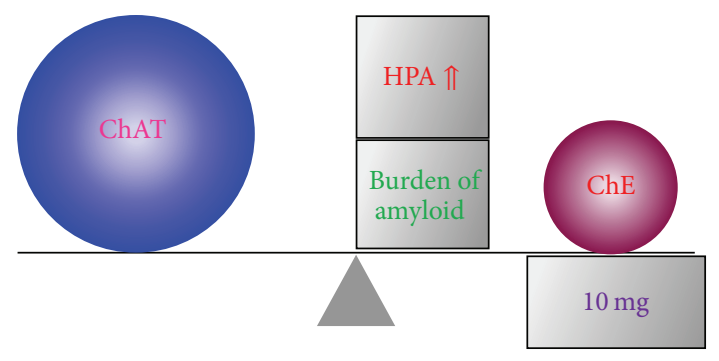

(b)

FIGURE 6: ChEI enhances ACh and enhanced ACh compensates for other AA inserts besides ACh downregulation. We prescribe three main ChEIs-donepezil, galantamine, and rivastigmine - to patients with mild or moderate stage AD. For donepezil, $5 \mathrm{mg}$ doses are allowed in Japan (a). Of these ChEIs (including generic medicines), only Aricept is permitted for LBD patients in Japan (since September 2014). In general, the dose of Aricept is $10 \mathrm{mg}$ for LBD (10 mg of donepezil is permitted only for severe AD patients in Japan) (b). We believe that the difference in required dosage between $\mathrm{AD}$ and $\mathrm{LBD}$ is because there is no other $\mathrm{AA}$ insert in $\mathrm{AD}$ besides ACh downregulation. However, another AA insert (e.g., HPA axis hyperactivity) is present in LBD, so it is important to upregulate ACh to compensate for this AA insert. AA: anticholinergic activity, ACh: acetylcholine, AD: Alzheimer's disease, ChEI: cholinesterase inhibitor, HPA axis: hypothalamic-pituitaryadrenal axis, and LBD: Lewy body disease. $5 \mathrm{mg}$ (in (a)): $5 \mathrm{mg}$ doses of donepezil; $10 \mathrm{mg}$ (in (b)): $10 \mathrm{mg}$ doses of Aricept (donepezil). This figure is reproduced from Konishi et al. [24] with the permission of Karger Publishers, Basel, Switzerland.

toxicity who complained of restlessness. Administration of $2 \mathrm{mg}$ of physostigmine, which enhances ACh by acting as a cholinesterase inhibitor (ChEI), immediately alleviated the symptoms. This case report appears to confirm our previous view that when the anticholinergic toxicity is caused by an exogenous factor (e.g., prescribed medicine), the mechanism of AA appearance depends on the endogenous system that is directly related to the ACh downregulation. Indeed, Flacker and Lipsitz [7] and Plaschke et al. [8] also commented that AA is caused by endogenous factors. We speculate that prescribed medication, physical illness, and mental stress induce HPA axis hyperactivity, which causes AA in persons whose ACh system is downregulated or overloaded, such as the elderly and patients with $\mathrm{AD}$ or LBD. Therefore, we prescribe ChEI to patients with a suspected anticholinergic spectrum disorder. ChEI enhances ACh and enhanced ACh compensates for other AA inserts besides ACh downregulation. Three main ChEIs-donepezil, galantamine, and rivastigmine-are prescribed to patients with mild or moderate stage AD. In Japan, $5 \mathrm{mg}$ doses of donepezil are allowed (Figure 6(a)). Of these ChEIs (including generic medicines), only Aricept ${ }^{\circledR}$ is permitted for LBD patients in Japan (since September 2014). In general, the dose of Aricept is $10 \mathrm{mg}$ for LBD (10 mg of donepezil is permitted only for severe $\mathrm{AD}$ patients in Japan) (Figure 6(b)). We believe that the difference in required dosage between $\mathrm{AD}$ and $\mathrm{LBD}$ is because there is no AA insert in $\mathrm{AD}$ other than $\mathrm{ACh}$ downregulation, whereas another $\mathrm{AA}$ insert is involved in LBD, such as HPA axis hyperactivity. Therefore, it is important to upregulate $\mathrm{ACh}$ to compensate for this AA insert. As for delirium, we can confirm that ChEI exerts positive and sudden effects. ChEI should thus be prescribed to patients with these spectrum disorders to abolish AA by upregulating ACh. Indeed delirium is considered to be related to downregulation of ACh [33-35] and AA [36-38] and ChEIs are favorable for delirium [39-41]. Therefore, delirium is suggestive of "anticholinergic spectrum disorders." We consider that this concept is enlarged to $\mathrm{AD}$ and LBD.

\section{Abbreviations}

$\begin{array}{ll}\text { AA: } & \text { Anticholinergic activity } \\ \text { ACh: } & \text { Acetylcholine } \\ \text { AD: } & \text { Alzheimer's disease } \\ \text { ChEI: } & \text { Cholinesterase inhibitor } \\ \text { HPA axis: } & \text { Hypothalamic-pituitary-adrenal axis } \\ \text { LBD: } & \text { Lewy body disease } \\ \text { SAA: } & \text { Serum anticholinergic activity. }\end{array}$

\section{Competing Interests}

Koji Hori has received lecture fees from Eisai Co. Ltd., Pfizer Japan Inc., Novartis Pharma KK, Daiichi Sankyo Inc., Ono Pharmaceutical Co. Ltd., Janssen Pharmaceutical KK, Yoshitomi Yakuhin Co., Meiji Seika Pharma Co. Ltd., and Mitsubishi Tanabe Pharma Co. Koji Hori has received funding from Eisai Co. Ltd., Daiichi Sankyo Inc., Ono Pharmaceutical Co. Ltd., Dainippon Sumitomo Pharm Co. Ltd., Eli Lilly Japan KK, and Shionogi \& Co. Ltd. Mitsugu Hachisu has received funding from Astellas Pharma Inc., Meiji Seika Pharma Co. Ltd., Dainippon Sumitomo Pharm Co. Ltd., Eli Lilly Japan KK, and Shionogi \& Co. Ltd. and has received lecture fees from Meiji Seika Pharma Co. Ltd. and Mitsubishi Tanabe Pharma Co.

\section{References}

[1] L. Tune and J. T. Coyle, "Serum levels of anticholinergic drugs in treatment of acute extrapyramidal side effects," Archives of General Psychiatry, vol. 37, no. 3, pp. 293-297, 1980.

[2] T. Sunderland, P. N. Tariot, R. M. Cohen, H. Weingartner, E. A. Mueller III, and D. L. Murphy, "Anticholinergic sensitivity in patients with dementia of the Alzheimer type and age-matched controls: a dose-response study," Archives of General Psychiatry, vol. 44, no. 5, pp. 418-426, 1987.

[3] O. J. Thienhaus, A. Allen, J. A. Bennett, Y. M. Chopra, and F. P. Zemlan, "Anticholinergic serum levels and cognitive 
performance," European Archives of Psychiatry and Clinical Neuroscience, vol. 240, no. 1, pp. 28-33, 1990.

[4] J. L. Cummings, "Cholinesterase inhibitors: a new class of psychotropic compounds," The American Journal of Psychiatry, vol. 157, no. 1, pp. 4-15, 2000.

[5] A. W. Lemstra, P. Eikelenboom, and W. A. van Gool, "The cholinergic deficiency syndrome and its therapeutic implications," Gerontology, vol. 49, no. 1, pp. 55-60, 2003.

[6] L. Tune, S. Carr, E. Hoag, and T. Cooper, "Anticholinergic effects of drugs commonly prescribed for the elderly: potential means for assessing risk of delirium," The American Journal of Psychiatry, vol. 149, no. 10, pp. 1393-1394, 1992.

[7] J. M. Flacker and L. A. Lipsitz, "Serum anticholinergic activity changes with acute illness in elderly medical patients," The Journals of Gerontology Series A: Biological Sciences and Medical Sciences, vol. 54, no. 1, pp. M12-M16, 1999.

[8] K. Plaschke, J. Kopitz, J. Mattern, E. Martin, and P. Teschendorf, "Increased cortisol levels and anticholinergic activity in cognitively unimpaired patients," Journal of Neuropsychiatry and Clinical Neurosciences, vol. 22, no. 4, pp. 433-441, 2010.

[9] K. Hori, K. Konishi, R. Akita et al., "Proposal of endogenous anticholinergic hypothesis in Alzheimer disease," Japanese Journal of Neuropsychopharmacology, vol. 33, no. 3, pp. 117-126, 2013 (Japanese).

[10] K. Hori, K. Konishi, M. Tani et al., "Serum anticholinergic activity: a possible peripheral marker of the anticholinergic burden in the central nervous system in Alzheimer's disease," Disease Markers, vol. 2014, Article ID 459013, 7 pages, 2014.

[11] Y. Kitajima, K. Hori, K. Konishi et al., "A review of the role of anticholinergic activity in Lewy body disease and delirium," Neurodegenerative Diseases, vol. 15, no. 3, pp. 162-167, 2015.

[12] K. Hori, K. Konishi, K. Watanabe et al., "Influence of anticholinergic activity in serum on clinical symptoms of Alzheimer's disease," Neuropsychobiology, vol. 63, no. 3, pp. 147-153, 2011.

[13] I.-C. Chien, J.-H. Hsu, S.-H. Bih et al., "Prevalence, correlates, and disease patterns of antipsychotic use in Taiwan," Psychiatry and Clinical Neurosciences, vol. 62, no. 6, pp. 677-684, 2008.

[14] A. Wood-Mitchell, I. A. James, A. Waterworth, A. Swann, and C. Ballard, "Factors influencing the prescribing of medications by old age psychiatrists for behavioural and psychological symptoms of dementia: a qualitative study," Age and Ageing, vol. 37 , no. 5, pp. 547-552, 2008.

[15] P. L. McGeer and E. G. McGeer, "The inflammatory response system of brain: implications for therapy of Alzheimer and other neurodegenerative diseases," Brain Research Reviews, vol. 21, no. 2, pp. 195-218, 1995.

[16] W. H. Frey II, C. R. Emory, M. E. Wiebenga et al., "Inhibitor of antagonist binding to the muscarinic receptor is elevated in Alzheimer's brain," Brain Research, vol. 655, no. 1-2, pp. 153-160, 1994.

[17] T. R. Bernik, S. G. Friedman, M. Ochani et al., "Pharmacological stimulation of the cholinergic antiinflammatory pathway," The Journal of Experimental Medicine, vol. 195, no. 6, pp. 781-788, 2002.

[18] J. G. Mabley, P. Packer, and C. Szabo, "Activation of the cholinergic antiinflammatory pathway reduces ricin-induced mortality and organ failure in mice," Molecular Medicine, vol. 15, no. 5-6, pp. 166-172, 2009.

[19] P. G. Nazarov, I. B. Krylova, N. R. Evdokimova, G. I. Nezhinskaya, and A. A. Butyugov, "C-reactive protein: a pentraxin with anti-acetylcholine activity," Life Sciences, vol. 80, no. 24-25, pp. 2337-2341, 2007.
[20] A. Fisher, “Therapeutic strategies in Alzheimer's disease: M1 muscarinic agonists," Japanese Journal of Pharmacology, vol. 84, no. 2, pp. 101-112, 2000.

[21] C. K. Jones, A. E. Brady, A. A. Davis et al., "Novel selective allosteric activator of the M1 muscarinic acetylcholine receptor regulates amyloid processing and produces antipsychotic-like activity in rats," Journal of Neuroscience, vol. 28, no. 41, pp. 10422-10433, 2008.

[22] E. K. Perry, L. Kilford, A. J. Lees, D. J. Burn, and R. H. Perry, "Increased Alzheimer pathology in Parkinson's disease related to antimuscarinic drugs," Annals of Neurology, vol. 54, no. 2, pp. 235-238, 2003.

[23] C.-J. Lu and L. E. Tune, "Chronic exposure to anticholinergic medications adversely affects the course of Alzheimer disease," The American Journal of Geriatric Psychiatry, vol. 11, no. 4, pp. 458-461, 2003.

[24] K. Konishi, K. Hori, H. Tomioka et al., "Donepezil abolishes anticholinergic activity in a patient with amnesia," Pharmacology, vol. 91, no. 1-2, pp. 86-91, 2013.

[25] J. Young and S. K. Inouye, "Delirium in older people," The British Medical Journal, vol. 334, no. 7598, pp. 842-846, 2007.

[26] E. Nederhof, K. Marceau, E. A. Shirtcliff, P. D. Hastings, and A. J. Oldehinkel, "Autonomic and adrenocortical interactions predict mental health in late adolescence: The TRAILS Study," Journal of Abnormal Child Psychology, vol. 43, no. 5, pp. 847-861, 2015.

[27] G. S. Masson, A. R. Nair, R. B. Dange, P. P. Silva-Soares, L. C. Michelini, and J. Francis, "Toll-like receptor 4 promotes autonomic dysfunction, inflammation and microglia activation in the hypothalamic paraventricular nucleus: role of endoplasmic reticulum stress," PLoS ONE, vol. 10, no. 3, Article ID e0122850, 2015.

[28] V. M. Miller, R. A. Kenny, A. E. Oakley, R. Hall, R. N. Kalaria, and L. M. Allan, "Dorsal motor nucleus of vagus protein aggregates in Lewy body disease with autonomic dysfunction," Brain Research, vol. 1286, pp. 165-173, 2009.

[29] I. G. McKeith, D. W. Dickson, J. Lowe et al., "Diagnosis and management of dementia with Lewy bodies: third report of the DLB Consortium," Neurology, vol. 65, no. 12, pp. 1863-1872, 2005.

[30] K. Hori, K. Konishi, H. Tanaka et al., "Pharmacotherapy for behavioral and psychological symptoms in Alzheimer's disease," Brain Disorders and Therapies, vol. 2, no. 2, article 106, 2013.

[31] L. Han, J. V. Agostini, and H. G. Allore, "Cumulative anticholinergic exposure is associated with poor memory and executive function in older men," Journal of the American Geriatrics Society, vol. 56, no. 12, pp. 2203-2210, 2008.

[32] M. S. Yilmaz, B. Işık, M. Ongar et al., "Delirium due to Datura stramoniun ingestion: a case report," Advances in Research, vol. 2, no. 10, pp. 523-527, 2014.

[33] P. T. Trzepacz, "Is there a final common neural pathway in delirium? Focus on acetylcholine and dopamine," Seminars in Clinical Neuropsychiatry, vol. 5, no. 2, pp. 132-148, 2000.

[34] T. T. Hshieh, T. G. Fong, E. R. Marcantonio, and S. K. Inouye, "Cholinergic deficiency hypothesis in delirium: a synthesis of current evidence," Journals of Gerontology, Series A: Biological Sciences and Medical Sciences, vol. 63, no. 7, pp. 764-772, 2008.

[35] A. Sfera, M. Cummings, and C. Osorio, "Non-neuronal acetylcholine: the missing link between sepsis, cancer, and delirium?" Frontiers in Medicine, vol. 2, article 56, 2015. 
[36] J. R. Mach Jr., M. W. Dysken, M. Kuskowski, E. Richelson, L. Holden, and K. M. Jilk, "Serum anticholinergic activity in hospitalized older persons with delirium: a preliminary study," Journal of the American Geriatrics Society, vol. 43, no. 5, pp. 491495, 1995.

[37] C. Mussi, R. Ferrari, S. Ascari, and G. Salvioli, "Importance of serum anticholinergic activity in the assessment of elderly patients with delirium," Journal of Geriatric Psychiatry and Neurology, vol. 12, no. 2, pp. 82-86, 1999.

[38] L. E. Tune, "Serum anticholinergic activity levels and delirium in the elderly," Seminars in Clinical Neuropsychiatry, vol. 5, no. 2, pp. 149-153, 2000.

[39] A. Baraka and S. Harik, "Reversal of central anticholinergic syndrome by galanthamine," The Journal of the American Medical Association, vol. 238, no. 21, pp. 2293-2294, 1977.

[40] S. P. Wengel, W. H. Eoccaforte, and W. J. Burke, "Donepezil improves symptoms of delirium in dementia: implications for future research," Journal of Geriatric Psychiatry and Neurology, vol. 11, no. 3, pp. 159-161, 1998.

[41] L. Stocker, L. Jellestad, J. Jenewein, and S. Boettger, "Challenges in the management of delirium: a case of augmentation with donepezil following inadequate response and adverse effects with risperidone," Psychiatria Danubina, vol. 27, no. 1, pp. 6466, 2015. 


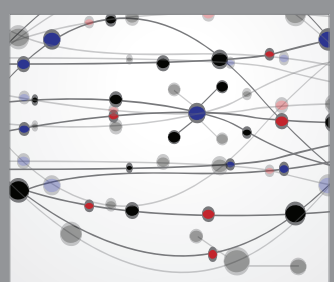

The Scientific World Journal
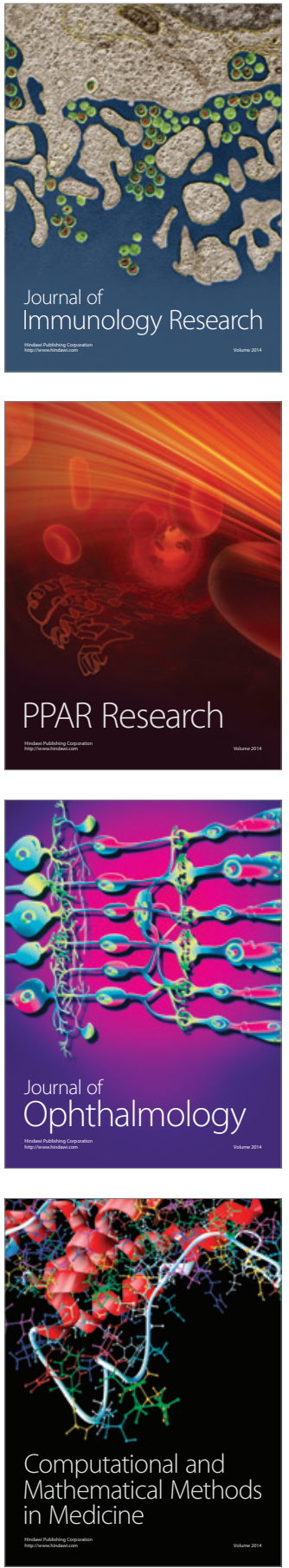

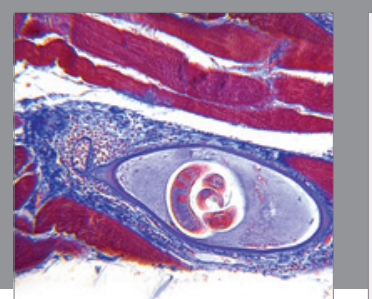

Gastroenterology Research and Practice

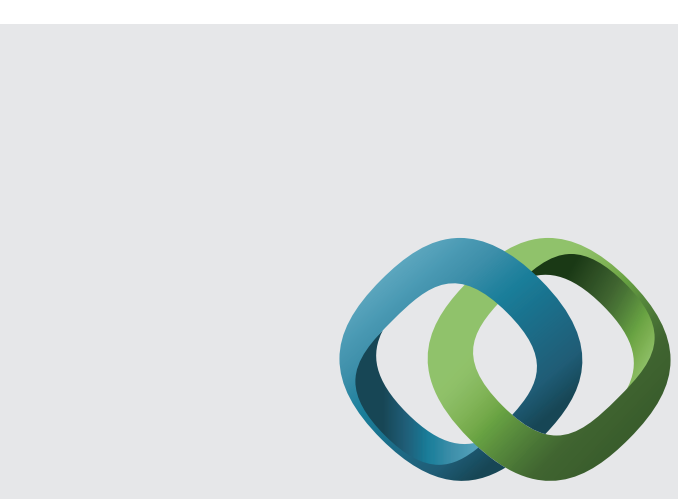

\section{Hindawi}

Submit your manuscripts at

http://www.hindawi.com
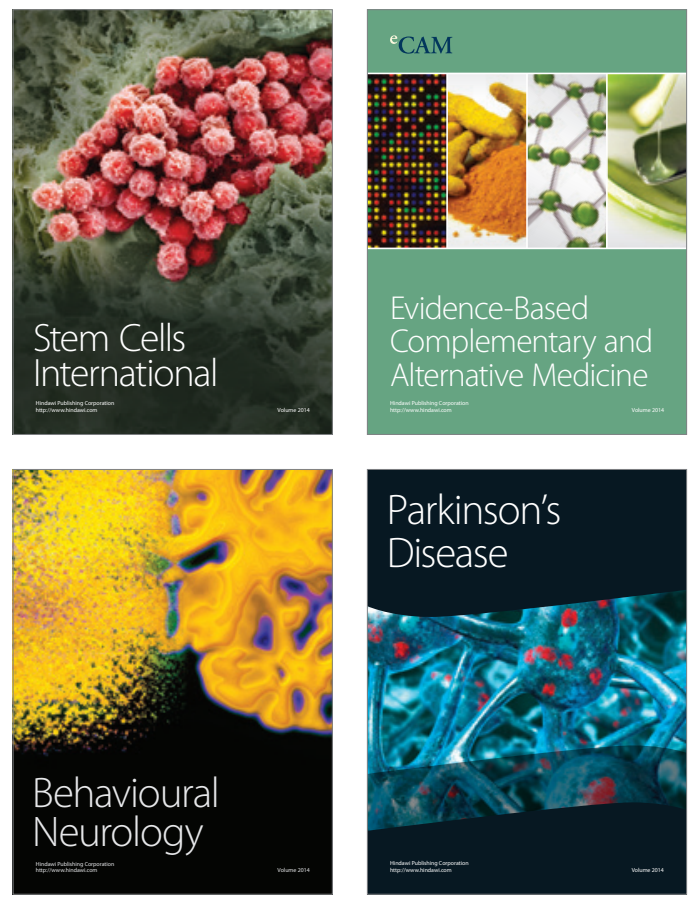
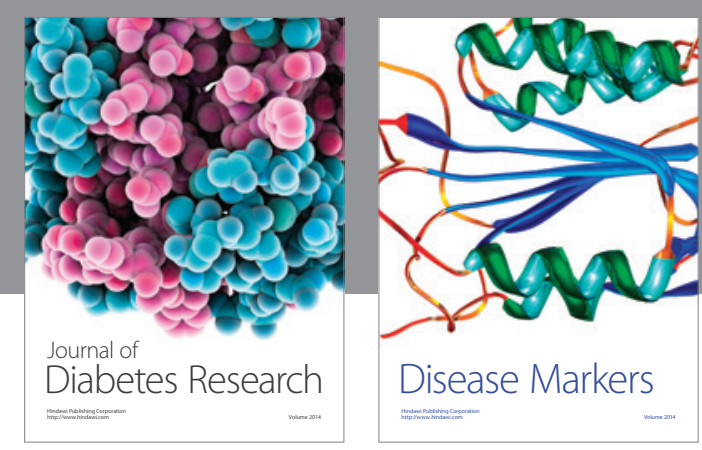

Disease Markers
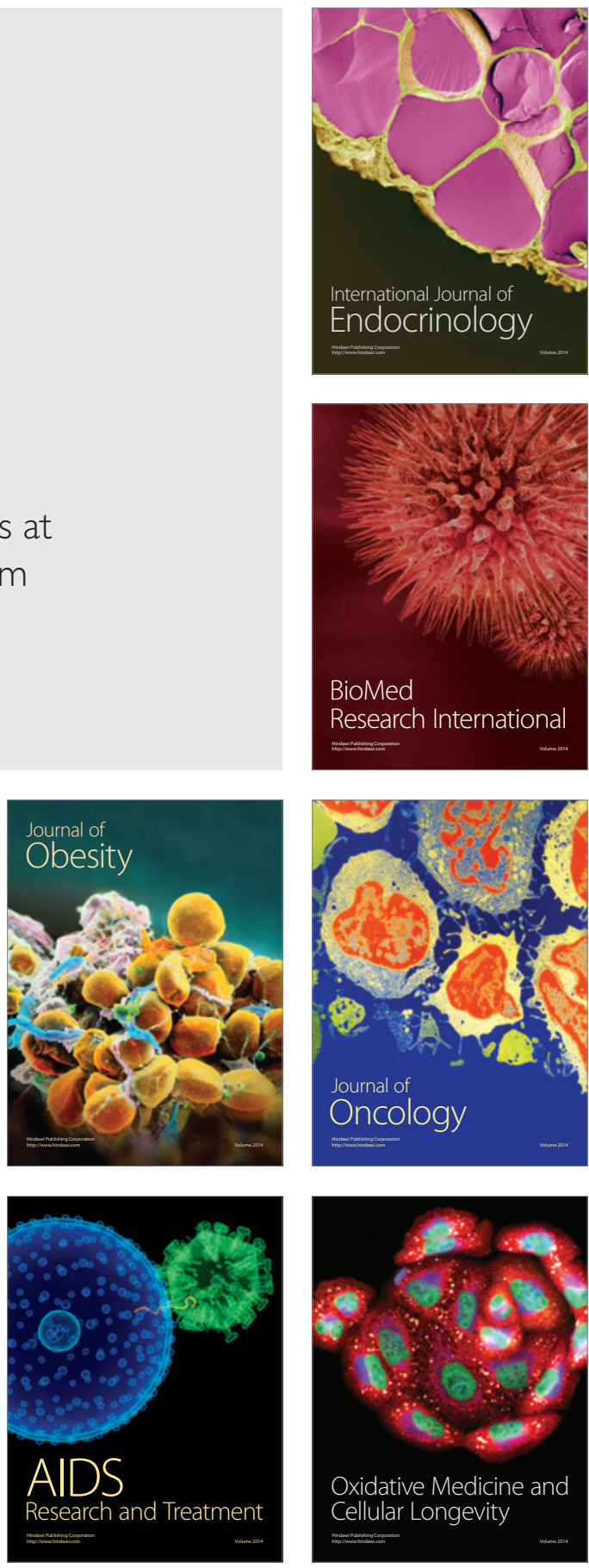